\title{
Mitochondrial AtMTM1 and AtMTM2 are required for MnSOD activation, stress response, and ion homeostasis
}

\author{
Shu-Hsuan Hu${ }^{1}$, Ya-Chen Huang ${ }^{1}$, Shu-Fan Lin ${ }^{1}$, Chien-Hsun Huang ${ }^{1}$, Wen-Yu Kuo ${ }^{1}$, and \\ Tsung-Luo Jinn ${ }^{1}$ \\ ${ }^{1}$ National Taiwan University
}

June 17,2020

\begin{abstract}
Yeast manganese (Mn) trafficking transporter for mitochondrial Mn-containing superoxide dismutase (MnSOD), yMTM1, belongs to mitochondrial carrier family (MCF) and is crucial for yeast MnSOD (ySOD2) activation. Arabidopsis AtMTM1 and AtMTM2 are homologs of yMTM1 and share conserved MCF motif sequence. We confirmed that AtMTM1 and AtMTM2 interacted with AtMnSOD (AtMSD1) in mitochondria and recovered ySOD2 activity in yMTM1-mutant cells. The redundant AtMTM1 and AtMTM2 have different gene expression patterns in tissues and methyl viologen (MV)-induced oxidative stress and also responded to most metal stresses along with AtMSD1. Bioassay revealed the contrasting root phenotype in microRNAmediated AtMTM1 mutant (mtm1-i) and AtMTM2-null mutant (mtm2) under MV stress, and Mn supplement complemented the root lengths in single and mtm1-i mtm2-double mutants. We found decreased MnSOD activity was accompanied by increased FeSOD activity in double mutant. Transient expression of chloroplast-destined AtMSD1 highlighted that unidentified factors participated in AtMSD1 activation. Besides, the exogenous-expressed AtMSD1 activity was decreased in double mutant, and inductively coupled plasma optical emission spectrometry results showed that AtMTM1 and AtMTM2 involved in Mn and Fe homeostasis with a reciprocal regulation. Overall, AtMTM1 and AtMTM2 are important for MnSOD metalation and ion homeostasis, and their physiological regulations may stretch across mitochondria and chloroplasts.
\end{abstract}

\section{KEYWORDS:}

Metallochaperone, Metalloprotein, Mitochondrial Carrier Family, Mn Trafficking Transporter, Reactive Oxygen Species, Superoxide Dismutase

\section{INTRODUCTION}

Harmful reactive oxygen species (ROS) contain non-free radicals and free radicals that are crucially regulated by the scavenger superoxide dismutases (SODs) during plant development and metabolism. Superoxide radicals are generated by metabolic processes of respiratory and photosynthetic electron transport chains in mitochondria and chloroplasts, and under high light, heat, drought, salt, and oxidative stress (Apel \& Hirt, 2004; Fridovich, 1975). Superoxide radicals are membrane-impermeable and rapidly damage nearby cell components, thus SOD enzymes catalyze the dismutation of the toxic superoxide radicals to oxygen and hydrogen peroxide, and defend against ROS (Halliwell, 1994).

SODs are metalloenzymes, and the corresponding cofactors are transition metal ions $(\mathrm{Cu}, \mathrm{Fe}, \mathrm{Mn}$, or $\mathrm{Ni})$ that accept or donate an electron during the dismutation process. Specially, SOD enzyme activation requires a metallochaperone or transporter that captures and loads the metal ion into the target SOD apoprotein. Most eukaryotes harbor both MnSOD and CuZnSOD. Prokaryotes and plants also contain FeSOD. NiSOD is present inStreptomyces (Alscher, Erturk, \& Heath, 2002; Choudhury et al., 1999; Fink \& Scandalios, 2002). Arabidopsis MnSOD (AtMSD1) locates in mitochondria (Bowler, Camp, Montagu, \& Inzé, 1994), FeSODs contain chloroplastic FSD1, FSD2, and FSD3. The stromal FSD1 conferred the mainly detectable FeSOD 
activity (Kuo, Huang, \& Jinn, 2013a; Kuo et al., 2013b). CuZnSODs contain cytosolic CSD1, chloroplastic CSD2, and peroxisomal CSD3 (Kliebenstein, Monde, \& Last, 1998).

The post-translational incorporation of Mn cofactor into yeast MnSOD (ySOD2) apoprotein involves the Mn trafficking transporter for mitochondrial MnSOD (yMTM1), which belongs to the mitochondrial carrier family (MCF) (Luk, Carroll, Baker, \& Culotta, 2003). MnSOD locates in the organelle of mitochondria in yeast and Arabidopsis, and also at the thylakoid membrane in some species of green and blue-green algae (Kanematsu \& Asada, 1979; Kanematsu, Okayasu, \& Kurogi, 2012; Okada, Kanematsu, \& Asada, 1979; Regelsberger et al., 2002). To date, the Mn-related chaperone in mitochondria or chloroplast has not been fully elucidated. The chloroplast chaperonin 20 (CPN20) functions as the Fe chaperone for FeSOD activation (Kuo et al., 2013a; Kuo et al., 2013b). Yeast copper chaperone for SOD1 (CCS) incorporates Cu cofactor into CuZnSOD apoprotein (ySOD1), and this CCS-dependent pathway also occurs in human and Arabidopsis (Casareno, Waggoner, \& Gitlin, 1998; Chu et al., 2005; Culotta, Yang, \& O'Halloran, 2006; Rae, Torres, Pufahl, \& O'Halloran, 2001). The CCS-independent CuZnSOD activation pathway was found in Arabidopsis cytoplasm and peroxisome, and glutathione was involved (Carroll et al., 2004; Huang, Kuo, \& Jinn, 2012a; Huang, Kuo, Weiss, \& Jinn, 2012b).

Arabidopsis mitochondria contain the highly conserved MnSOD that congregates as a homotetramer, and each monomer harbors only one Mn cofactor (Pilon, Ravet, \& Tapken, 2011; Sevilla, López-Gorgé, \& del Río, 1982). Among all species of SOD, MnSOD is responsible for protecting the respiratory machinery in the mitochondrial matrix (Bowler et al., 1994; Fridovich, 1975). MnSOD expression is more constant than FeSOD and CuZnSOD under high light, ozone, and UV-B exposure (Kliebenstein et al., 1998). Overexpressing $M n S O D$ could increase stress tolerance in transgenic tobacco and Arabidopsis (Slooten et al., 1995; Van Camp et al., 1994; Wang, Ying, Chen, \& Wang, 2004). By contrast, decreased MnSOD expression may cause root growth inhibition and affect mitochondrial redox homeostasis in Arabidopsis (Morgan et al., 2008). On the other hand, CuZnSOD and FeSOD enzymes protect chloroplasts against superoxide radicals, and their activities are easily affected by $\mathrm{Cu}$ level via $\mathrm{Cu}$ transporters of chloroplastic envelop-localized $\mathrm{P}$-type ATPase PAA1 and thylakoid membrane-localized PAA2. Specially, FSD1 and CSD2 genes are affected by stromal $\mathrm{Cu}$ delivery with a reciprocal regulation (Abdel-Ghany et al., 2005a; Abdel-Ghany, Muller-Moule, Niyogi, Pilon, \& Shikanai, 2005b).

In E. coli, MnSOD and FeSOD share a well-conserved protein folds and have conserved residues for metal binding (Pugh \& Fridovich, 1985; Wintjens et al., 2004). Incorrect metal insertion altered SOD reduction potential, and the enzyme had trouble scavenging superoxide radicals (Miller, 2012; Vance \& Miller, 2001). $\mathrm{Mn}$ and Fe are interchangeable between MnSOD and FeSOD, and cause metal ion misincorporation, but neither Fe-substituted MnSOD nor Mn-substituted FeSOD is active (Ganini, Petrovich, Edwards, \& Mason, 2015; Meier, Barra, Bossa, Calabrese, \& Rotilio, 1982).

In yeast, ySOD2 protein cannot be activated in the cytosol. Mn cofactor is inserted into the newly synthesized ySOD2 polypeptides via the mitochondrial carrier protein yMTM1, thus the insertion of Mn into ySOD2 apoprotein is connected to the importing process in mitochondria (Luk, Yang, Jensen, Bourbonnais, \& Culotta, 2005). In yMTM1 -mutant $(\psi \mu \tau \mu 1 \Delta)$ cells, inactivated ySOD2 is associated with the disrupted mitochondrial Fe homeostasis. When the Fe-S cluster biogenesis pathway was blocked, Fe for Fe-S cluster diverted to ySOD2 and caused Fe misincorporation, but the role of yMTM1 in Fe-S cluster maturation has not been verified (Naranuntarat, Jensen, Panicni, Penner-Hahn, \& Culotta, 2009; M. Yang et al., 2006). In Arabidopsis, mitochondrial carrier protein AtMTM1 (At4g27940) has 32\% identity with yMTM1, and can recover ySOD2 activity in $\psi \mu \tau \mu 1 \Delta$ cells (Su et al., 2007).

Mn transporters for MnSOD activation are associated with Mn homeostasis in yeast and Arabidopsis. Mn homeostasis in yeast involves Mn transporters SMF1 and SMF2 in the natural resistance-associated macrophage protein (NRAMP) family (Chen et al., 1999; Luk \& Culotta, 2001). SMF1 is located at the plasma membrane of cell surface; SMF2 localizes in intracellular vesicles and functions for Mn trafficking among intracellular locations (Portnoy, Liu, \& Culotta, 2000).SMF1 mutation did not affect ySOD2 activity, but SMF2disruption caused cell-wide Mn starvation and loss of ySOD2 activity (Luk \& Culotta, 2001). 
Arabidopsis NRAMP family members NRAMP3 and NRAMP4 have homology with yeast SMF1 and SMF2, and are involved in the Mn-containing oxygen-evolving complex (OEC). OEC catalyzes the water-splitting reaction that produces oxygen and provides electrons for the photosynthetic electron transport chain (Goussias, Boussac, \& Rutherford, 2002; Nickelsen \& Rengstl, 2013). Disruption of vacuole-localized NRAMP3 and NRAMP4 decreased PSII amount in chloroplasts, but did not affect MnSOD activity in mitochondria (Allen, Kropat, Tottey, Del Campo, \& Merchant, 2007; Lanquar et al., 2010).

The system of Mn transportation in plants involves families of NRAMP, Zn-regulated transporter and Feregulated transporter-like protein (ZIP), yellow stripe-like protein (YSL), cation exchanger (CAX), calcium cation exchanger (CCX), cation diffusion facilitator/metal tolerance protein (CDF/MTP), vacuolar Fe transporter (VIT), and P-type ATPase (Socha \& Guerinot, 2014). MCF family member MTM1 is directly connected to MnSOD activation via transporting Mn cofactor into the MnSOD apoprotein. The essential Mn cofactor is also required for other metalloproteins such as oxalate oxidase, RNA polymerase, malic enzyme, and isocitrate dehydrogenase (Bashir, Rasheed, Kobayashi, Seki, \& Nishizawa, 2016; Marschner, 1995; Rostami \& Ahangar, 2013; Witholt, Gwiazda, \& Smith, 2000).

The MCF is a large family with 35 members in yeast and more than 50 genes in plants and humans, which is evolutionarily conserved for transporting the specific substrates and cofactors (Haferkamp \& Schmitz-Esser, 2012). MCF members have three homologous domains which fold into two transmembrane $\alpha$-helices, and contain the conserved mitochondrial energy transfer signature (METS), P-x-[DE]-x-[LIVAT]-[RK]-x-[LRH][LIVMFY]-[QGAIVM], on the matrix side (Millar \& Heazlewood, 2003; Robinson \& Kunji, 2006). Study of the evolution of MCF revealed a clustered phylogeny of AtMTM1 and AtMTM2, and their amino acid sequences have high homology (Palmieri, Pierri, Grassi, Nunes-Nesi, \& Fernie, 2011).

In this research, we clarified the similar complementary effect of AtMTM1 and AtMTM2 for ySOD2 activation in $\psi \mu \tau \mu 1 \Delta$ cells, and revealed their regulations under methyl viologen (MV)-mediated oxidative stress. We confirmed that an evolutionarily conserved mechanism for MnSOD activation is remained in Arabidopsis chloroplast as in some species of algae. In addition, we strengthened the impact of AtMTM1 and AtMTM2 on $\mathrm{Mn}$ and Fe ion homeostasis.

\section{MATERIALS AND METHODS}

\section{Plants and growth condition}

Arabidopsis (Arabidopsis thaliana) accession Columbia-0 (Col) was used in this study as the wild-type (WT) control. Mutants ofmtm1-1 (SALK 023286), mtm1-2 (SALK 054287C), mtm2-1(SALK 005166), mtm2-2 (SALK 103984), and mtm2-3 (SALK 025071) were obtained from the Arabidopsis Biological Resource Center (ABRC, Ohio State University). Plants were grown in growth chambers at $22-24^{\circ} \mathrm{C}$ under a 16 -h light $/ 8$-h dark cycle at 80-100 $\mu \mathrm{mol} \mathrm{m} \mathrm{m}^{-2} \mathrm{~s}^{-1}$. For root length assay, sterile seeds were placed on solid half-strength Murashige and Skoog basal medium (1/2 MS; Sigma M5519) (Murashige \& Skoog, 1962) containing $1 \%$ Suc and $0.8 \%$ phytagel (Sigma). Transgenic plants of AtMTM2 -promoter::GUS with pCAMBIA-1391Z vector, and $35 S$ ::AtMTM1 -overexpression (OE) and $35 S$ ::AtMTM2 -OE with pCAMBIA-3300 vectors in Arabidopsis Col background were generated by Agrobacterium tumefaciensGV3101-mediated transformation and the floral dip method (Clough \& Bent, 1998). For post-translational regulation, metal stress, and metal ion homeostasis assays, two-week-old seedlings were transferred to the stressed medium under the normal 16-h light/8-h dark condition as described above.

\section{Yeast strains and growth condition}

Yeast (Saccharomyces cerevisiae) used in this study contained the WT BY4741 (MATa, $\eta \imath 3 \Delta 1, \lambda \epsilon v 2 \Delta 0$, $\mu \epsilon \tau 15 \Delta 0, v \rho a 3 \Delta 0), \psi \sigma o \delta 2 \Delta($ sod2::kanMX4), and $\psi \mu \tau \mu 1 \Delta$ (mtm1::LEU2 MC101) mutants. Yeasts were incubated at $30^{\circ} \mathrm{C}$ under aerobic condition (Brachmann et al., 1998). Enriched yeast extract-peptone-dextrose (YPD) media was supplemented with $2 \%$ Glc, and synthetic dextrose (SD) medium was used to culture yeast strains. For yeast expression, all cDNAs were cloned into the yeast expression vector pADNS. Yeast transformation involved the lithium acetate procedure (Gietz \& Schiestl, 1991), and transformants were 
selected on minimal SD media. Yeast was incubated at $30^{\circ} \mathrm{C}$ under the aerobic condition without shaking, and G418 was used at $200 \mu \mathrm{g} \mathrm{mL} \mathrm{m}^{-1}$ to maintain $y S O D 2$ and $y M T M 1$ deletion mutants of $\psi \sigma o \delta 2 \Delta$ and $\psi \mu \tau \mu 1 \Delta$ . For plate assay, yeast cultures were diluted serially from $A_{600}=1$ to $10^{-4}$. Yeast transformants were plated without or with $\mathrm{MV}$, and incubated at $30^{\circ} \mathrm{C}$ under the aerobic condition for $10 \mathrm{~d}$.

\section{Protein extraction and SOD activity analysis}

Yeast lysates were extracted by the glass bead lysis method (Culotta et al., 1997). Arabidopsis leaf extracts were prepared with a grinding buffer of $150 \mathrm{mM}$ Tris $(\mathrm{pH} 7.2)$. The homogenate was centrifuged at 12,000 $\mathrm{rpm}$ at $4^{\circ} \mathrm{C}$ for $10 \mathrm{~min}$. Total protein was separated on $10 \%$ non-denaturing polyacrylamide gel, and the photochemical method of in-gel activity assay was used to analyze SOD activity (Beauchamp \& Fridovich, 1971; Kliebenstein et al., 1998; Kuo, Huang, Shih, \& Jinn, 2013c). The SOD activity was quantified by analyzing activity gel with the UVP ChemStudio PLUS imaging system (Analytik Jena US), and verified by $\mathrm{KCN}$ and $\mathrm{H}_{2} \mathrm{O}_{2}$ treatments (Kuo et al., 2013c). KCN is an inhibitor of CuZnSOD, and $\mathrm{H}_{2} \mathrm{O}_{2}$ inhibits both CuZnSOD and FeSOD. MnSOD activity is not affected by either chemical (Balzan, Bannister, Hunter, \& Bannister, 1995; Chen, Weng, Ho, Cheng, \& Lai, 2000; Kuo et al., 2013c).

Genomic DNA and RNA preparations, cDNA synthesis, and quantitative real-time PCR (qPCR)

Genomic DNA was extracted for genotyping as previously reported (Edwards et al., 1991). Total RNA was prepared with TRIZOL reagent (Invitrogen) and involved with TURBO DNA-free Kit (Applied Biosystems). cDNA synthesis was performed with the Ready-To-Go Kit (GE Healthcare Life Sciences). PCR primers were designed by Primer3 (http://primer3.ut.ee). qPCR reactions with iQ SYBR Green Supermix (Bio-Rad) were analyzed by MyiQ thermocycler (Bio-Rad). Data were analyzed with the iQ5 optical system (Bio-Rad), and normalized to the internal control AtPP2AA3(PP2A; At1g13320) (Czechowski, Stitt, Altmann, Udvardi, \& Scheible, 2005).

\section{Localization and bimolecular fluorescence complementation (BiFC) assays}

The full-length cDNA fragments of AtMTM1, AtMTM2, andAtMSD1 were PCR amplified and cloned into the pCR8/GW/TOPO vector (Invitrogen). Constructs were recombined into the pEarleyGate101 vector for subcellular localization, or into the pEarleyGate201-YN and pEarleyGate202-YC for BiFC analysis (Lu et al., 2010). Four-week-old plants were used for protoplast preparation and transfection, as described in the earlier report (Yoo, Cho, \& Sheen, 2007). $2 \times 10^{4}$ protoplasts and $15 \mu \mathrm{g}$ DNA were used for transfection, and YFP signals were observed by using a TCS SP5 confocal microscope (Leica).

\section{AtMTM2 histochemical analysis and generation of AtMTM1-miRNA mutants}

The 2-kb potential AtMTM2 promoter activity during plant development was analyzed by GUS staining (Weigel \& Glazebrook, 2002). The 21mer artificial microRNA (miRNA) of AtMTM1 was cloned into pRS300 vector which contains the miR319a precursor (Schwab, Ossowski, Riester, Warthmann, \& Weigel, 2006). The automated design of artificial miRNAs was according to the Web app (http://wmd3.weigelworld.org/cgibin/webapp.cgi). The final PCR fragment was subcloned into pPZP200GB vector for constructing microRNA-mediated AtMTM1 suppression lines.

\section{Constructs of organelle-destined AtMSD1}

The deletion of the mitochondrial transit peptide of AtMSD1 resulted in cytosol-localized AtMSD1. The mitochondrial transit peptide of AtMSD1 that was replaced by the chloroplast transit peptide of CSD2 (Huang et al., 2012b) became chloroplast-destined AtMSD1. Besides, a tag of 15-amino acids or 3xFLAG was fused to the C-terminus of AtMSD1 to distinguish the exogenous-expressed AtMSD1 from endogenous AtMSD1.

Inductively coupled plasma optical emission spectrometry (ICP-OES)

ICP-OES (Perkin Elmer Optima 5300 DV) was conducted to evaluate metal ion content, as described 
previously (Lahner et al., 2003; Lin et al., 2009; Yang, Perry, Ciani, Pandian, \& Schmidt, 2008). Two-weekold seedlings were removed from 1/2 MS medium and incubated with an additional $100 \mu \mathrm{M} \mathrm{MnCl} 2$ for 24 $\mathrm{h}$ with agitation. Aerial parts (shoots) and roots tissues were dried at $60^{\circ} \mathrm{C}$ for $3 \mathrm{~d}$ and an amount of 0.1 g sample was analyzed by ICP-OES. Spinach and tomato leaves (SRM-1570a and SRM-1573a; US National Institute of Standards and Technology) were the standard references.

\section{Statistical analysis}

Three biological and technical repeats were conducted. Independent Student's $t$-test (two-tailed) was used to compare difference for all tests. $P<0.05$ was considered statistically significant.

\section{Primers and accession numbers}

Primers used and accession numbers are listed in Supplemental Table S1 .

\section{RESULTS}

\section{Arabidopsis MTM1 and MTM2 are homologs of yeast MTM1}

The sequence alignment of AtMTM2, AtMTM1, and yMTM1 showed high similarity (Supplemental Figure S1) . Each protein sequence consists of three tandemly homologous domains (blue underline), and each repeat domain contains two transmembrane $\alpha$-helices (yellow shading). Three copies of a 10-amino acid sequence motif (I, II, and III) known as the METS are the structural features of MCF carriers. Besides, they share the conserved substrate Mn-binding site (red double-headed arrow).

Protein BLAST (http://blast.ncbi.nlm.nih.gov/Blast.cgi) revealed AtMTM1 has 32\% protein sequence identity with yMTM1; AtMTM2 has 34\% protein identity with yMTM1 and 59\% identity with AtMTM1. Sequence alignment and motif analysis revealed AtMTM1 and AtMTM2 could be homologs of the yeast MCF protein yMTM1. As noted, AtMTM1 was suggested as the yMTM1 homolog in Arabidopsis (Su et al., 2007).

\section{AtMTM1 and AtMTM2 localize in mitochondria and interact with AtMnSOD (AtMSD1)}

The subcellular localization of AtMTM1 and AtMTM2 was examined with a yellow fluorescent protein (YFP) fused to their C-terminal ends, and expressed in Arabidopsis protoplasts (Figure 1A). The fluorescence signals of both AtMTM1-YFP and AtMTM2-YFP were merged well with MitoTracker, a mitochondriaspecific dye. The results confirmed the presence of both proteins in mitochondria, as for yMTM1-green fluorescent protein (GFP) and AtMTM1-GFP in earlier reports (Luk et al., 2003; Su et al., 2007).

Bimolecular fluorescence complementation (BiFC) assay was conducted to examine protein-protein interactions among AtMTM1, AtMTM2, and AtMSD1(Figure 1B). The reconstituted YFP signals revealed that AtMSD1 interacted with AtMTM1 and AtMTM2 independently, as well as the interaction of AtMTM1 and AtMTM2 (Supplemental Figure S2), indicating AMTM1 and AtMTM2 contribute to AtMSD1 activity. Control experiments (Huang, Niu, Yang, \& Jinn, 2016; Walter et al., 2004) were shown in Supplemental Figure S3 .

\section{AtMTM1 and AtMTM2 are functional homologs of yMTM1}

To analyze the complementary effect of AtMTM1 and AtMTM2 for yMTM1 in yeast MnSOD activation, we transformed AtMTM1 and AtMTM2in yMTM1 -mutant $(\psi \mu \tau \mu 1 \Delta)$ cells (Figure 2) .

Yeast wild-type (WT) cells contained one activity band for CuZnSOD (ySOD1) and two bands for MnSOD (ySOD2) and residual ySOD2 activities remained in $\psi \mu \tau \mu 1 \Delta$ cells (Figure 2, lanes 1, 2), as reported earlier (Luk et al., 2003). We showed ySOD2 activities were recovered when $\psi \mu \tau \mu 1 \Delta$ cells were transformed with AtMTM1 or AtMTM2 (Figure 2, lanes 3, 4), indicating AtMTM1 and AtMTM2 are functional homologs of yMTM1. Moreover, the ySOD2 activities were not increased by co-transformation of AtMTM1 and AtMTM2 (Figure 2, lane 5), implying the synergistic effect did not occur in $\psi \mu \tau \mu 1 \Delta$ cells.

In conclusion, AtMTM1 and AtMTM2 transformations recovered ySOD2 activities in $\psi \mu \tau \mu 1 \Delta$ cells, which was similar toyMTM1 transformation (Supplemental Figure S4), indicating they shared similar ability 
for ySOD2 activation.

\section{AtMSD1 activation depends on AtMTM1 participation}

To investigate the dependence of Mn cofactor and AtMTM1 for AtMSD1 activation, we co-expressed AtMTM1 and AtMSD1 inySOD2 -mutant ( $\psi \sigma o \delta 2 \Delta)$ cells with Mn supplement(Figure 3) .

The ySOD2 activities increased clearly in WT cells under $100 \mu \mathrm{M} \mathrm{MnSO}_{4}$ treatment (Figure 3A, lane 2) , indicating the importance of Mn cofactor as previously reported by Luk et al. (2003). Compared with the AtMSD1 transformation (Figure 3A , lanes 5, 6), co-expression of AtMTM1 and AtMSD1 increased AtMSD1 activities markedly in $\psi \sigma o \delta 2 \Delta$ cells(Figure 3A, lanes 5, 7), reflecting AtMTM1 participated in AtMSD1 activation. Mn supplementation had no substantial effect on AtMSD1 activity (Figure 3A, lanes $6,8)$.

To examine the protective roles of AtMTM1 and AtMSD1 under oxidative stress, we transformed $\psi \sigma \sigma \delta 2 \Delta$ cells and challenged to methyl viologen (MV), an oxidative-stress inducer (Figure 3B).AtMSD1 transformation restored $\psi \sigma o \delta 2 \Delta$ cells to the WT phenotype with MV treatment (Figure 3B, lanes 3, 4) , and AtMSD1 co-expressed with AtMTM1 revealed similar protection ability (Figure $\mathbf{3 B}$, lanes $\mathbf{5}, \mathbf{6}$ ) . The result implied that a conserved effect of yMTM1 for AtMSD1 activation, as AtMTM1 for ySOD2 activation (Supplemental Figure S4) .

The mitochondrial localization of AtMSD1 in $\psi \sigma o \delta 2 \Delta$ cells was confirmed by subcellular fractionation and immunoblotting(Supplemental Figure S5A). AtMSD1 was colocalized with a mitochondrial marker porin (Supplemental Figure S5A, lanes 1, 3) . A cytosolic marker phosphoglycerate kinase (PGK) was used as control (Supplemental Figure S5A, lanes 1, 2). The exogenous-expressed AtMSD1 activity and protein in $\psi \sigma o \delta 2 \Delta$ cells were detected by in-gel SOD activity assay and immunoblotting(Supplemental Figure S5B, lanes 3, 4), respectively. The AtMSD1 activity was confirmed with KCN treatment which inhibits ySOD1 activity (Supplemental Figure S5B, lanes 6, 7).

Expressions of AtMTM1, AtMTM2, and AtMSD1 during development

The expression of AtMTM1 was previously analyzed in transgenic AtMTM1 -promoter:: GUS plants (Su et al., 2007). In this study, we generated AtMTM2 -promoter:: GUS plants to establish AtMTM2 expression profile (Supplemental Figure S6), and clarified AtMTM1, AtMTM2, and AtMSD1 mRNA expressions by qPCR (Figure 4 ) .

The 2-kb potential AtMTM2 promoter region was fused with the reporter gene GUS , and transgenic plants were stained for GUS activity (Supplemental Figure S6) . AtMTM2 expression was clear in hypocotyl, root, and cotyledon in 5-d-old young seedlings(Supplemental Figure S6A to C) . AtMTM2 expression was also detected in 15-d-old trichomes, rosette leaf, cauline leaf(Supplemental Figure S6D to F), and flower organs of sepal, petal, anther, pollen, stigma, and embryo in siliques(Supplemental Figure S6G to I) .

We further compared the gene expression levels of AtMTM1,AtMTM2, and AtMSD1 in root, rosette leaf, cauline leaf, flower stem, flower, and silique by qPCR (Figure 4). AtMTM2 had higher expression than AtMTM1 in rosette and cauline leaves. AtMSD1 expression was ubiquitously higher, but lower in flower and silique. Notably, both AtMTM1 and AtMTM2 had profound expressions in flower.

Post-transcriptional regulation of AtMSD1 and expressions of AtMTM1, AtMTM2, and $A t M S D 1$ under a shock of oxidative stress

To check the regulation of AtMSD1 in response to oxidative stress, we treated 2-week-old seedlings with 0.1 to $10 \mu \mathrm{M} \mathrm{MV}$ for a shock of 12 or $24 \mathrm{~h}$ oxidative stress and examined MnSOD activity. We also compared AtMTM1 , AtMTM2, and AtMSD1 gene expression profiles under MV stress (Figure 5) .

The MnSOD activity in WT increased gradually with the increased dose of MV for $24 \mathrm{~h}$ by in-gel activity assay (Figure 5A, top), but MnSOD protein level remained similar by immunoblotting (Figure 5A, 
bottom), reflecting the phenomena of post-transcriptional regulation of MnSOD activity.

Specially, we observed the earlier and higher expression for AtMTM2 than AtMTM1 in response to $5 \mu \mathrm{M}$ MV for $12 \mathrm{~h}$ treatment by qPCR (Figure 5B, top). AtMSD1 and mitochondria oxidative stress-responsive maker gene AtAOX1A were up-regulated (Figure 5B, bottom) . Cytoplasmic oxidation-responsive marker genes AtAPX1 and AtCAT1 were used as references, and they also responded to MV stress at the early stage (Supplemental Figure S7) . Overall, the post-transcriptional MnSOD activity in WT under MV stress corresponded with the up-regulated AtMTM1, AtMTM2, and AtMSD1 genes.

\section{Expressions of AtMTM1, AtMTM2, and AtMSD1 under metal stresses}

To elucidate the effect of metal stress on AtMTM1, AtMTM2 , and AtMSD1 gene regulations, we transferred 2-week-old seedlings to buffers containing $100 \mu \mathrm{M}$ of $\mathrm{Mn}\left(\mathrm{MnCl}_{2}\right), \mathrm{Fe}$ (Fe citrate), $\mathrm{Cu}\left(\mathrm{CuSO}_{4}\right), \mathrm{Zn}\left(\mathrm{ZnSO}_{4}\right)$, $\mathrm{Mg}\left(\mathrm{MgSO}_{4}\right)$, and $\mathrm{Ca}\left(\mathrm{CaCl}_{2}\right)$ for $24 \mathrm{~h}$ with gentle shaking (Figure 6) .

Compared to the unstressed condition, AtMTM1, AtMTM2 , and AtMSD1 expressions responded positively to most metal stresses by qPCR. The $100 \mu \mathrm{M}$ Mn treatment caused no significant effect on AtMTM1 expression. AtMTM2 was markedly responsive to $\mathrm{Cu}$ stress compared with AtMTM1 and AtMSD1 . Generally, all three genes were up-regulated synchronically under metal stresses.

\section{Characterization of Arabidopsis mtm1, mtm1-i, andmtm2 mutant lines}

To investigate the physiological functions of AtMTM1 and AtMTM2, we characterized T-DNA insertion mutants of mtm1-1 and -2, and microRNA-mediated AtMTM1- mutant (mtm1-i) lines (Supplemental Figures S8, 9) . Besides, homozygous T-DNA insertion lines of mtm2-1 , -2 , and-3 were analyzed (Supplemental Figure S10) .

In mtm1-1 and -2 mutants, T-DNA is inserted in the 3'-UTR and 5'-UTR of the AtMTM1 gene, respectively (Supplemental Figure S8A) . Homozygous lines were confirmed by genotyping. RT-PCR and qPCR revealed both mtm1-1 and -2 are knock-down mutants, and the $m t m 1-1$ is a $45 \%$ knock-down mutant compared with the WT(Supplemental Figure S8B) .

We screened five stable T4 lines of $m t m 1-i$ mutant(Supplemental Figure S9A), and the two lowest expression lines remained about 20\% AtMTM1 expression (Supplemental Figure S9B, mtm1-i-3 and -5). The mtm1-i-3 mutant remained unaffected MnSOD and FeSOD activities compared with the WT(Supplemental Figure S9C) .

In mtm2-1 mutant, T-DNA is inserted in the last exon of theAtMTM2 coding region (Supplemental Figure S10A) . Genotyping, RT-PCR, and qPCR confirmed mtm2-1 is a null mutant. MnSOD and FeSOD activities in mtm2-1 are similar to the WT(Supplemental Figure S10B). In mtm2-2 and -3mutants, T-DNA is inserted in the intron, but RT-PCR assay showed the expression of AtMTM2 was not majorly affected(Supplemental Figs. S10A, C) .

In the following study, we examined the redundant function and synergistic effect of AtMTM1 and AtMTM2 by using $m t m 1-i-3$ (referred as mtm1-i ) and mtm2-1 (referred as mtm2) mutants.

\section{Dependency between AtMTM1 and AtMTM2 in adapting to a shock of oxidative stress}

To check the dependency between AtMTM1 and AtMTM2 in response to oxidative stress, two-week-old seedlings of mtm1-iand mtm2 were treated with 1 to $10 \mathrm{nM} \mathrm{MV}$ for a shock of $12 \mathrm{~h}$ (Figure 7) .

The expression of AtMTM2 in mtm1-i knock-down mutant was not affected compared with the WT under 1 to $10 \mathrm{nM}$ MV stress(Figure 7A). However, AtMTM1 expression in mtm2knock-out mutant was significantly up-regulated compared with the WT in adapting to 1 and $5 \mathrm{nM}$ MV stress (Figure 7B), reflecting redundant AtMTM1 and AtMTM2 genes have different dependency.

Phenotypes of $m t m 1-i$ and $m t m 2$ mutants in adaption to long-term MV stress 
Altered root elongation is an indicator of oxidative stress during plant development (Tsukagoshi, 2012). For long-term oxidative stress, we monitored the root growth of $m t m 1-i$ and $m t m 2$ seedlings in various MVcontaining plates for 5 to 15 d (Figure 8; Supplemental Figure S11). The treatment period and MV concentration varied depending on the mutants.

In 8-d-old and 15-d-old seedlings, mtm1-i had significantly inhibited root growth compared with the WT under 10 to $30 \mathrm{nM}$ MV treatments (Figure 8A; Supplemental Figure S11A, B) . In 10-d-old and 5-d-old seedlings, mtm2 had significantly longer root length than the WT under 1 to $10 \mathrm{nM}$ MV treatments (Figure 8B; Supplemental Figure S11C). Taken together, root lengths of 5 to 15-d-old mtm1-i and mtm2 seedlings showed the contrasting phenotypes compared with the WT under MV stress, implying redundant AtMTM1 and AtMTM2 genes have different responses toward oxidative stress.

Moreover, one-month-old $m t m 1-i$ and $m t m 2$ plants both exhibited the early-flowering phenotypes compared with the WT under normal growth condition (Supplemental Figure S11D), indicating AtMTM1 and AtMTM2 play a role in this pathway.

Synergistic effects of AtMTM1 and AtMTM2 in mtm1-i mtm2-double mutants

In order to discover the synergistic effect of AtMTM1 and AtMTM2 genes, we crossed mtm1-i and mtm2 to generate the $m t m 1-i$ mtm2 -double mutant (Figure 9) .

Two stable lines of mtm1-i mtm2 -double mutant (\#1 and\#2) were screened and confirmed by qPCR (Figure 9A), and the lowest expression line (\#1) was focused. Six independent siblings of the mtm1$i$ mtm2-\#1 revealed wobbling MnSOD and FeSOD activities (Figure 9B). Compared with the WT, the relative SOD activity analysis in three siblings of mtm1-i mtm2-\#1 showed the decreased MnSOD activity was accompanied by increased FeSOD activity(Figure 9C) . In addition, mtm1-i mtm2-\#1 -double mutant retained the early-flowering phenotype compared with the WT(Figure 9D) and single mutants (Supplemental Figure S12), indicating AtMTM1 and AtMTM2 have synergistic effects on MnSOD activity and early-flowering phenotype.

Rescue of mtm1-i, mtm2, and mtm1-i mtm2root phenotype under Mn supplemental condition

To clarify the defective metal regulation in transporter mutants, researchers usually applied metal supplementation and observed phenotypic complementation (Eisenhuta et al., 2018; Schneider et al., 2016). The $1 / 2 \mathrm{MS}$ basal salt mixture contained $50 \mu \mathrm{M} \mathrm{Mn}$ and other required nutrients. The root lengths of $m t m 1-i$, mtm2 , and mtm1-i mtm2-\#1 (referred as mtm1-i mtm2) were monitored on 1/2 MS plate without or with additional 10 to $500 \mu \mathrm{M} \mathrm{MnCl} 2$ for $6 \mathrm{~d}$ (Figure 10).

Without additional Mn treatment, we observed the root length of 6-d-old $m t m 1$ - $i$ seedlings was shorter; the $m t m 2$ and $m t m 1-i m t m 2$ were longer than the WT. The root length of $m t m 1-i$ was recovered on additional 10 and $50 \mu \mathrm{M}$ Mn-containing plates compared with the WT. The extra $500 \mu \mathrm{M} \mathrm{Mn} \mathrm{supplement} \mathrm{rescued} \mathrm{the}$ root length to the WT in all single and double mutants. Overall, the additional Mn supplement revealed the complementary effect of the root length in single and double mutants.

\section{Overexpression of AtMTM1 and AtMTM2 had no effect on MnSOD activity}

We overexpressed AtMTM1 and AtMTM2 with a CaMV35S promoter(Supplemental Figure S13). Three independent lines of AtMTM1 -overexpression (OE) and AtMTM2 -OE showed enhanced gene expressions by RT-PCR (Supplemental Figure S13A, B) . Nonetheless, MnSOD activity remained unchanged in AtMTM1 -OE and AtMTM2 -OE plants compared with the WT (Supplemental Figure S13C), implying the saturated level of MnSOD activity retained in these overexpression lines as the WT.

\section{Chloroplast-destined AtMSD1 activation is independent of AtMTM1 and AtMTM2}

To seek other candidates for MnSOD activation, we generated cytosol-destined and chloroplast-destined AtMSD1, and transfected these constructs into the WT and mtm1-i mtm2 protoplasts for comparison(Figures 11, 12). 
The destinations of cytosol-localized AtMSD1 ( $\Delta$-TP-AtMSD1) and chloroplast-localized AtMSD1 (Chl-TPAtMSD1) were confirmed with a reporter YFP and analyzed by confocal microscopy (Figure 11A) . Besides, the exogenous-expressed AtMSD1-Tag and endogenous MnSOD activities could be distinguished by in-gel SOD activity assay and immunoblotting (Figure 11B, lanes 1, 2). Particularly, the chloroplast-destined AtMSD1 was activated but not the cytosol-localized AtMSD1 (Figure 11B, lanes 3, 4) . Overall, we found the exogenous expressed AtMSD1 activation inside chloroplast was independent of mitochondrial AtMTM1 and AtMTM2, and unclarified chloroplastic factors contributed to chloroplast-destined MSD1 metalation.

We also created a modified AtMSD1 with a 3xFLAG tag, and transfected this construct into the WT and mtm1-i mtm2 -double mutant protoplasts (Figure 12). The exogenous-expressed AtMSD1-3xFLAG activity and protein were confirmed by in-gel SOD activity assay and immunoblotting (Supplemental Figure S14), respectively. Notably, the exogenous expressed AtMSD1-3xFLAG activity was lower in $m$ tm1$i$ mtm2 than the WT with transfection of $10 \mu \mathrm{g}$ DNA for $16 \mathrm{~h}$ (Figure 12, lanes 3, 6), implying AtMTM1 and AtMTM2 participated in AtMSD1 activation.

Ion contents of mtm1-i, mtm2, and mtm1-i mtm2 mutant plants in response to $\mathrm{MnCl}_{2}$ treatment

To investigate the impact of metal ion homeostasis through AtMTM1 and AtMTM2 that have been classified as the mitochondrial carriers (Picault, Hodges, Palmieri, \& Palmieri, 2004), we applied $100 \mu \mathrm{M} \mathrm{MnCl}_{2}$ treatment on mtm1-i, mtm2, andmtm1-i mtm2 mutants, and used ICP-OES to measure metal ion contents. The index of metal retention ability represents the ratio of the ion content in $\mathrm{Mn}$ treatment to that in control (Figure 13, Supplemental Figure S15) .

The mtm1-i , mtm2, and mtm1-i mtm2 mutant lines had decreased Mn content in root compared with the WT under Mn treatment, and mtm2 had lower Mn content before treatment (Figure 13A, top) . Specially, the mtm1-i mtm2 -double mutant had decreased Mn retention ability by 1.3-fold in root compared with the WT. On the contrary, all three mutants had increased Mn content in shoot compared with the WT under Mn treatment (Figure 13A, bottom), implying the discrepancy of Mn regulation was in root and shoot via AtMTM1 and AtMTM2.

Altered Fe homeostasis with a reciprocal trend was also observed in root and shoot of these single and double mutants under Mn treatment(Figure 13B). Fe retention ability was reduced significantly in root of these mutants, even in the control of mtm1-i mtm2 -double mutant (Figure 13B, top). On the contrary, all three mutants had increased Fe content in shoot before and after treatments. Particularly, the double mutant had increased Fe retention ability by 1.3-fold in shoot compared with the WT (Figure 13B, bottom) .

Taken together, the reciprocal regulation of $\mathrm{Mn}$ and Fe contents in root and shoot were detected in single and double mutants, especially inmtm1-i mtm2 -double mutant, indicating $\mathrm{Mn}$ and Fe homeostasis are closely regulated by AtMTM1 and AtMTM2. Besides, the fluctuations of other metal contents and retention abilities of $\mathrm{Ca}, \mathrm{Mg}, \mathrm{Zn}, \mathrm{K}$, and $\mathrm{Na}$ were observed in single or double mutants before or after Mn treatment(Supplemental Figure S15), implying AtMTM1 and AtMTM2 may extensively affect cellular ion homeostasis.

\section{DISCUSSION}

Mitochondria matrix communicates with the cytosol via the MCF proteins that are evolutionarily conserved. Arabidopsis MCF proteins contain three homologous repeated domains of about 100 amino acids, and each domain has a characteristic motif (Kunji, 2004; Picault et al., 2004). MCF proteins exist as homodimers, and the monomer represents one functional entity (Kunji \& Crichton, 2010). These carrier proteins locate at the mitochondrial inner membrane and catalyze specific transport of inorganic ions, cofactors, metabolites, and nucleotides into the mitochondrial matrix (Haferkamp \& Schmitz-Esser, 2012).

Phylogenetic analysis showed that the high homology of MCF genesAtMTM1 and AtMTM2 are clustered (Palmieri et al., 2011), and characterization of yMTM1 functional-like gene AtMTM1 was reported (Su et al., 2007). In this study, we characterized its homolog AtMTM2, investigated the redundant function of 
AtMTM1 and AtMTM2, and looked for other candidates for AtMSD1 activation. For the physiological roles of AtMTM1 and AtMTM2, we provided evidence of their mitochondrial localization, interaction with AtMSD1, and the same complementation ability for ySOD2 activation in $\psi \mu \tau \mu 1 \Delta$ cells(Figs. 1, 2) . Co-transformation of AtMTM1 increased AtMSD1 activity, and AtMSD1 enhanced the survival rate in $\psi \sigma o \delta 2 \Delta$ cells under MV stress (Figure 3). This phenomenon is consistent with the concept that the mitochondrial MCF protein yMTM1 has a positive effect on increased ySOD2 activity (Luk et al., 2003). Our data strengthened the importance of AtMTM1 and AtMTM2 for AtMSD1 activity and cell growth in yeast and Arabidopsis.

Expression analysis of Arabidopsis MCF proteins revealed that paired carriers have distinct specificities during plant growth and abiotic stress (Catoni et al., 2003; Hoyos et al., 2003; Maia et al., 1998; Watanabe, Nakazono, Tsutsumi, \& Hirai, 1999). For the discrepancy of AtMTM1 and AtMTM2, we found AtMTM1 and AtMTM2 had enhanced expressions in flower tissue during development, but AtMTM2 played a dominant role compared withAtMTM1 under MV stress (Figures 4, 5). The previous study mentioned MnSOD activity increased under $\mathrm{Cu}$ and Cd stresses (Drażkiewicz, Skórzyńska-Polit, \& Krupa, 2007). In this study, we applied multiple metal-mediated oxidative stresses, not onlyAtMSD1 but AtMTM1 and AtMTM2 gene expressions increased synchronously (Figure 6). For comparison of $m t m 1-i$ knock-down and $m t m 2$ knockout single mutants, onlymtm2 mutant had significantly up-regulated AtMTM1 gene expression under MV stress, and these two single mutants had a contrasting effect of root growth compared with the WT in response to MV stress (Figures $\mathbf{7 , 8 )}$. All these results confirmed the redundant AtMTM1 and AtMTM2 genes have different capabilities toward stresses.

Notably, the decreased MnSOD protein accompanied by increased FeSOD protein level was found in $M n S O D$ -antisense Arabidopsis (Morgan et al., 2008). In our study, the mtm1-i mtm2 -double mutant also had decreased MnSOD activity with increased FeSOD activity (Figure 9). We proposed the physiological regulations of AtMTM1 and AtMTM2 stretch across mitochondria and chloroplasts, and may have an impact on flowering in Arabidopsis. Moreover, the Mn supplement rescued the root lengths of mtm1-i, mtm2, and mtm1-i mtm2 mutants to the WT, and revealed the complementary effect of Mn transportation (Figure 10) .

To look for other candidates for the post-translational process of MnSOD activation, we generated modified AtMSD1 constructs of cytosolic and chloroplastic versions, and only the chloroplast-destined MSD1 can be activated in WT protoplasts (Figure 11) . We proposed the organelle-specific Mn transporters or chaperones for chloroplastic-destined MSD1 activation exist in Arabidopsis. This phenomenon is similar to the model that $\mathrm{Mn}$ insertion is associated with the mitochondrial importing and the protein folding process (Luk et al., 2005). Taken together, we concluded the insertion of Mn cofactor via the membrane transporter is necessary for MnSOD activation in Arabidopsis.

In addition to mitochondria, MnSOD is present in the thylakoids of some prokaryotic and eukaryotic algae (Kanematsu \& Asada, 1979; Okada et al., 1979; Regelsberger et al., 2002). In this study, we found the factors for the exogenous-expressed AtMSD1 metalation still exist in Arabidopsis chloroplasts, implying chloroplastic Mn transporters may participate in chloroplast-destined AtMSD1 activation that could be evolutionarily conserved. The chloroplast transporter CMT1 mediated Mn transportation (Eisenhuta et al., 2018; Zhang et al., 2018), and the PAM71/CCHA1 regulated Mn and Ca homeostasis in chloroplast (Schneider et al., 2016; Wang et al., 2016). Both inner envelope-localized CMT1 and thylakoid membrane-localized PAM71/CCHA1 are crucial for the biogenesis of $\mathrm{Mn}$ cluster $\mathrm{Mn}_{4} \mathrm{CaO}_{5}$ during photosynthesis (Krieger-Liszkay \& Thomine, 2018). Taken together, the roles of CMT1, PAM71/CCHA1, Mn cluster-related factors, and released Mn from PSII for AtMSD1 activation in chloroplast remain to be investigated.

Moreover, we found the exogenous-expressed AtMSD1 activity inmtm1-i mtm2 -double mutant was expressed at a lower level compared with the WT (Figure 12). Thus, we proposed the defectiveAtMTM1 and AtMTM2 cause the blockage of Mn transportation and affect AtMSD1 metalation synergistically. Several factors for mitochondrial MnSOD inactivation have also been studied in yeast. Fe-S cluster biogenesis genes GRX5 and SSQ1 involved in ySOD2 inactivation by disrupting mitochondrial Fe homeostasis in yeast 
(Naranuntarat et al., 2009). In Mn-deficient plants, the altered Mn content in root or shoot tissue was involved in the abnormal root growth, altered Fe homeostasis, and gene expressions (Alejandro et al., 2017; Rodríguez-Celma et al., 2016; Yang et al., 2008). yMTM1 facilitated the insertion of Mn cofactor for ySOD2 activation, but $\psi \mu \tau \mu 1 \Delta$ retained the normal Mn level in mitochondria (Luk et al., 2003). In our study, we found Mn transportation was defective inmtm1-i, mtm2, and mtm1-i mtm2 mutants, and both Mn and $\mathrm{Fe}$ contents in root and shoot were responsive to $\mathrm{Mn}$ treatment, especially in $m t m 1-i$ mtm2 -double mutant (Figure 13). We further proposed AtMTM1 and AtMTM2 transporters regulate Mn and Fe homeostasis with a reciprocal regulation. For the metal ion affinity, many metal transporters have multiple metal substrates, but the substrate specificities of AtMTM1 and AtMTM2 remain to be clarified.

In conclusion, we emphasized the physiological function of Arabidopsis mitochondrial carrier proteins AtMTM1 and AtMTM2 for MnSOD enzyme activation, and compared their gene expression profiles during development and oxidative stress. In addition to AtMTM1 and AtMTM2, we suggested the MnSOD importing process is associated with other transporters or chaperones that may exist in both mitochondria and chloroplasts. We confirmed AtMTM1 and AtMTM2 transporters regulate the intracellular Mn and Fe redox states, and the involved mechanism may cross organelles in cells.

\section{SUPPLEMENTAL DATA}

Supplemental Figure S1. Sequence alignment of AtMTM2, AtMTM1, and yMTM1.

Supplemental Figure S2 . Interaction of AtMTM1 and AtMTM2 in BiFC assay.

Supplemental Figure S3 . Control experiments for BiFC assay.

Supplemental Figure S4. AtMTM1, AtMTM2, andyMTM1 recovered ySOD2 activity in $\psi \mu \tau \mu 1 \Delta$ cells.

Supplemental Figure S5 . AtMSD1 localization and activity in $\psi \sigma o \delta 2 \Delta$ cells.

Supplemental Figure S6 . Histochemical analysis of AtMTM2 -promoter:: GUS expression.

Supplemental Figure S7 . Expressions of oxidation-responsive marker genes of cytoplasmic AtAPX1 and AtCAT1 under MV stress.

Supplemental Figure S8 . Characterization of T-DNA insertion lines of $m t m 1-1$ and -2 .

Supplemental Figure S9 . Characterization of $m t m 1-i$ lines.

Supplemental Figure S10 . Characterization of T-DNA insertion lines of mtm2-1 , -2, and -3 .

Supplemental Figure S11. Phenotypes of $m t m 1-i$ and $m t m 2$ seedlings in adaption to long-term MV stress.

Supplemental Figure S12 . Photograph of early-flowering phenotypes in mtm1-i, mtm2, and mtm1-i mtm2 plants.

Supplemental Figure S13. Characterization of AtMTM1 and AtMTM2 overexpression lines.

Supplemental Figure S14. AtMSD1-3xFLAG activity in WT protoplasts.

Supplemental Figure S15 . Metal contents and metal retention abilities of $\mathrm{Ca}, \mathrm{Mg}, \mathrm{Zn}, \mathrm{K}$, and $\mathrm{Na}$ in mtm1-i , mtm2, andmtm1-i mtm2 mutants under $\mathrm{MnCl}_{2}$ treatment.

\section{ACKNOWLEDGEMENTS}

We are grateful to Fang-Jen Lee for the gift of yeast BY4741, $\psi \sigma o \delta 2 \Delta$, and $\psi \mu \tau \mu 1 \Delta$ lines that were obtained from the Saccharomyces Genome Deletion Project, Daniel J. Kliebenstein for kindly providing the antisera of Arabidopsis MnSOD, and Kuo-Chen Yeh for helping ICP-OES analysis. We would like to thank Lynne Stracovsky for editing, Hui-Chen Wu for commenting the manuscript, and Pei-Chun Hsieh for technical support. We also appreciate the NTU Confocal Microscope Laboratory for performing fluorescence imaging. 


\section{REFERENCES}

Abdel-Ghany, S. E., Burkhead, J. L., Gogolin, K. A., Andrés-Colás, N., Bodecker, J. R., Puig, S., . . Pilon, M. (2005a). AtCCS is a functional homolog of the yeast copper chaperone Ccs1/Lys7. FEBS Letters, 11, $2307-2312$.

Abdel-Ghany, S. E., Muller-Moule, P., Niyogi, K. K., Pilon, M., \& Shikanai, T. (2005b). Two P-type ATPases are required for copper delivery in Arabidopsis thaliana chloroplasts. Plant Cell, 17, 1233-1251.

Alejandro, S., Cailliatte, R., Alcon, C., Dirick, L., Domergue, F., Correia, D., . . Curie, C. (2017). Intracellular distribution of manganese by the trans-Golgi network transporter NRAMP2 is critical for photosynthesis and cellular redox homeostasis. Plant Cell, 29, 3068-3084.

Allen, M. D., Kropat, J., Tottey, S., Del Campo, J. A., \& Merchant, S. S. (2007). Manganese deficiency in Chlamydomonas results in loss of photosystem II and MnSOD function, sensitivity to peroxides, and secondary phosphorus and iron deficiency. Plant Physiology, 143, 263-277.

Alscher, R. G., Erturk, N., \& Heath, L. S. (2002). Role of superoxide dismutases (SODs) in controlling oxidative stress in plants. Journal of Experimental Botany, 53, 1331-1341.

Apel, K., \& Hirt, H. (2004). Reactive oxygen species: metabolism, oxidative stress, and signal transduction. Annual Review of Plant Biology, 55, 373-399.

Balzan, R., Bannister, W. H., Hunter, G., \& Bannister, J. V. (1995). Escherichia coli iron superoxide dismutase targeted to the mitochondria of yeast cells protects the cells against oxidative stress. Proceedings of the National Academy of Sciences of the United States of America, 92, 4219-4223.

Bashir, K., Rasheed, S., Kobayashi, T., Seki, M., \& Nishizawa, N. K. (2016). Regulating subcellular metal homeostasis: The key to crop improvement. Frontiers in Plant Science, 1192.

Beauchamp, C., \& Fridovich, I. (1971). Superoxide dismutase: improved assays and an assay applicable to acrylamide gels. Analytical Biochemistry, 44, 276-287.

Bowler, C., Camp, W. V., Montagu, M. V., \& Inzé, D. (1994). Superoxide dismutase in plants. Critical Reviews in Plant Sciences, 13, 199-218.

Brachmann, C. B., Davies, A., Cost, G. J., Caputo, E., Li, J., Hieter, P., \& Boeke, J. D. (1998). Designer deletion strains derived from Saccharomyces cerevisiae S288C: a useful set of strains and plasmids for PCRmediated gene disruption and other applications. Yeast, 14, 115-132.

Carroll, M. C., Girouard, J. B., Ulloa, J. L., Subramaniam, J. R., Wong, P. C., Valentine, J. S., \& Culotta, V. C. (2004). Mechanisms for activating $\mathrm{Cu}$ - and Zn-containing superoxide dismutase in the absence of the CCS Cu chaperone Proceedings of the National Academy of Sciences of the United States of America, 101, 5964-5969.

Casareno, L. B., Waggoner, D., \& Gitlin, J. D. (1998). The copper chaperone CCS directly interacts with copper/zinc superoxide dismutase. Journal of Biological Chemistry, 273, 23625-23628.

Catoni, E., Schwab, R., Hilpert, M., Desimone, M., Schwacke, R., Flügge, U. I., . . Frommer, W. B. (2003). Identification of an Arabidopsis mitochondrial succinate-fumarate translocator. FEBS Letters, 534, 87-92.

Chen, J. R., Weng, C. N., Ho, T. Y., Cheng, I. C., \& Lai, S. S. (2000). Identification of the copper-zinc superoxide dismutase activity in Mycoplasma hyopneumoniae. Veterinary Microbiology, 73, 301-310.

Chen, X. Z., Peng, J. B., Cohen, A., Nelson, H., Nelson, N., \& Hediger, M. A. (1999). Yeast SMF1 mediates $\mathrm{H}(+)$-coupled iron uptake with concomitant uncoupled cation currents. Journal of Biological Chemistry, 274, 35089-35094. 
Choudhury, S. B., Lee, J. W., Davidson, G., Yim, Y. I., Bose, K., Sharma, M. L., . . Maroney, M. J. (1999). Examination of the nickel site structure and reaction mechanism in Streptomyces seoulensis superoxide dismutase. Biochemistry, 38, 3744-3752.

Chu, C. C., Lee, W. C., Guo, W. Y., Pan, S. M., Chen, L. J., Li, H. M., \& Jinn, T. L. (2005). A copper chaperone for superoxide dismutase that confers three types of copper/zinc superoxide dismutase activity in Arabidopsis. Plant Physiology, 139, 425-436.

Clough, S. J., \& Bent, A. F. (1998). Floral dip: a simplified method for Agrobacterium-mediated transformation of Arabidopsis thaliana. Plant Journal, 16, 735-743.

Culotta, V. C., Klomp, L. W., Strain, J., Casareno, R. L., Krems, B., \& Gitlin, J. D. (1997). The copper chaperone for superoxide dismutase. Journal of Biological Chemistry, 272, 23469-23472.

Culotta, V. C., Yang, M., \& O'Halloran, T. V. (2006). Activation of superoxide dismutases: putting the metal to the pedal. Biochimica et Biophysica Acta, 1763, 747-758.

Czechowski, T., Stitt, M., Altmann, T., Udvardi, M. K., \& Scheible, W. R. (2005). Genome-wide identification and testing of superior reference genes for transcript normalization in Arabidopsis. Plant Physiology, 139, $5-17$.

Drażkiewicz, M., Skórzyńska-Polit, E., \& Krupa, Z. (2007). The redox state and activity of superoxide dismutase classes in Arabidopsis thaliana under cadmium or copper stress. Chemosphere, 67, 188-193.

Edwards, K., Johnstone, C., \& Thompson, C. (1991). A simple and rapid method for the preparation of plant genomic DNA for PCR analysis. Nucleic Acids Research, 19, 1349.

Eisenhuta, M., Hoecker, N., Schmidt, S. B., Basgaran, R. M., Flachbart, S., Jahns, P., . . . Schneider, A. (2018). The plastid envelope CHLOROPLAST MANGANESE TRANSPORTER1 is essential for manganese homeostasis in Arabidopsis. Molecular Plant, 11, 955-969.

Fink, R. C., \& Scandalios, J. G. (2002). Molecular evolution and structure-function relationships of the superoxide dismutase gene families in angiosperms and their relationship to other eukaryotic and prokaryotic superoxide dismutases. Archives of Biochemistry and Biophysics, 399, 19-36.

Fridovich, I. (1975). Superoxide dismutases. Annual Review of Biochemistry, 44, 147-159.

Ganini, D., Petrovich, R. M., Edwards, L. L., \& Mason, R. P. (2015). Iron incorporation into MnSOD A (bacterial Mn-dependent superoxide dismutase) leads to the formation of a peroxidase/catalase implicated in oxidative damage to bacteria. Biochimica et Biophysica Acta, 1850, 1795-1805.

Gietz, R. D., \& Schiestl, R. H. (1991). Applications of high efficiency lithium acetate transformation of intact yeast cells using single-stranded nucleic acids as carrier. Yeast, 7, 253-263.

Goussias, C., Boussac, A., \& Rutherford, A. W. (2002). Photosystem II and photosynthetic oxidation of water: an overview. Philosophical Transactions of the Royal Society B, 357, 1369-1381.

Haferkamp, I., \& Schmitz-Esser, S. (2012). The plant mitochondrial carrier family: functional and evolutionary aspects. Frontiers in Plant Science, $3,2$.

Halliwell, B. (1994). Free radicals, antioxidants, and human disease: curiosity, cause, or consequence? Lancet, 344, 721-724.

Hoyos, M. E., Palmieri, L., Wertin, T., Arrigoni, R., Polacco, J. C., \& Palmieri, F. (2003). Identification of a mitochondrial transporter for basic amino acids in Arabidopsis thaliana by functional reconstitution into liposomes and complementation in yeast. Plant Journal, 33, 1027-1035.

Huang, C. H., Kuo, W. Y., \& Jinn, T. L. (2012a). Models for the mechanism for activating copper-zinc superoxide dismutase in the absence of the CCS Cu chaperone in Arabidopsis. Plant Signaling \& Behavior, $7,429-431$. 
Huang, C. H., Kuo, W. Y., Weiss, C., \& Jinn, T. L. (2012b). Copper chaperone-dependent and -independent activation of three copper-zinc superoxide dismutase homologs localized in different cellular compartments in Arabidopsis. Plant Physiology, 158, 737-746.

Huang, Y. C., Niu, C. Y., Yang, C. R., \& Jinn, T. L. (2016). The heat stress factor HSFA6b connects ABA signaling and ABA-mediated heat responses. Plant Physiology, 172, 1182-1199.

Kanematsu S, Asada K. 1979. Ferric and manganese superoxide dismutases in Euglena gracilis. Archives of Biochemistry and Biophysics 195, 535-545.

Kanematsu S, Okayasu M, Kurogi D. 2012. Occurrence of two types of Mn-superoxide dismutase in the green alga Spirogyra: cDNA cloning and characterization of genomic genes and recombinant proteins. Bulletin of Minamikyushu University 42A, 1-13.

Kanematsu, S., \& Asada, K. (1979). Ferric and manganese superoxide dismutases in Euglena gracilis. Archives of Biochemistry and Biophysics, 195, 535-545.

Kanematsu, S., Okayasu, M., \& Kurogi, D. (2012). Occurrence of two types of Mn-superoxide dismutase in the green alga Spirogyra: cDNA cloning and characterization of genomic genes and recombinant proteins. Bulletin of Minamikyushu University, 42A, 1-13.

Kliebenstein, D. J., Monde, R. A., \& Last, R. L. (1998). Superoxide dismutase in Arabidopsis: an eclectic enzyme family with disparate regulation and protein localization. Plant Physiology, 118, 637-650.

Krieger-Liszkay, A., \& Thomine, S. (2018). Importing manganese into the chloroplast: many membranes to cross. Molecular Plant, 11, 1109-1111.

Kunji, E. R. S. (2004). The role and structure of mitochondrial carriers. FEBS Letters, 564, 239-244.

Kunji, E.R. S., \& Crichton, P. G. (2010). Mitochondrial carriers function as monomers. Biochimica et Biophysica Acta, 1797, 817-831.

Kuo WY, Huang CH, Jinn TL. 2013a. Chaperonin 20 might be an iron chaperone for superoxide dismutase in activating iron superoxide dismutase (FeSOD). Plant Signaling \& Behavior, 8, e23074.

Kuo WY, Huang CH, Liu AC, Cheng CP, Li SH, Chang WC, Weiss C, Azem A, Jinn TL. 2013b. Chaperonin 20 mediates iron superoxide dismutase (FeSOD) activity independent of its co-chaperonin role in Arabidopsis chloroplasts. New Phytologist, 197, 99-110.

Kuo WY, Huang CH, Shih C, Jinn TL. 2013c. Cellular extract preparation for superoxide dismutase (SOD) activity assay. Bio-protocol 3, e811.

Kuo, W. Y., Huang, C. H., \& Jinn, T. L. (2013a). Chaperonin 20 might be an iron chaperone for superoxide dismutase in activating iron superoxide dismutase (FeSOD). Plant Signaling \& Behavior, 8, e23074.

Kuo, W. Y., Huang, C. H., Liu, A. C., Cheng, C. P., Li, S. H., Chang, W. C., . . . Jinn, T. L. (2013b). Chaperonin 20 mediates iron superoxide dismutase (FeSOD) activity independent of its co-chaperonin role in Arabidopsis chloroplasts. New Phytologist, 197, 99-110.

Kuo, W. Y., Huang, C. H., Shih, C., \& Jinn, T. L. (2013c). Cellular extract preparation for superoxide dismutase (SOD) activity assay. Bio-protocol, 3, e811.

Lahner, B., Gong, J., Mahmoudian, M., Smith, E. L., Abid, K. B., Rogers, E. E., . . . Salt, D. E. (2003). Genomic scale profiling of nutrient and trace elements in Arabidopsis thaliana. Nature Biotechnology, 21, 1215-1221.

Lanquar, V., Ramos, M. S., Lelievre, F., Barbier-Brygoo, H., Krieger-Liszkay, A., Kramer, U., \& Thomine, S. (2010). Export of vacuolar manganese by AtNRAMP3 and AtNRAMP4 is required for optimal photosynthesis and growth under manganese deficiency. Plant Physiology, 152, 1986-1999. 
Lin, Y. F., Liang, H. M., Yang, S. Y., Boch, A., Clemens, S., Chen, C. C., . . . Yeh, K. C. (2009). Arabidopsis IRT3 is a zinc-regulated and plasma membrane localized zinc/iron transporter. New Phytologist, 182, 392404.

Lu, Q., Tang, X., Tian, G., Wang, F., Liu, K., Nguyen, V., . . . Cui, Y. (2010). Arabidopsis homolog of the yeast TREX-2 mRNA export complex: components and anchoring nucleoporin. Plant Journal, 61, 259-270.

Luk, E. E., \& Culotta, V. C. (2001). Manganese superoxide dismutase in Saccharomyces cerevisiae acquires its metal co-factor through a pathway involving the Nramp metal transporter, Smf2p. Journal of Biological Chemistry, 276, 47556-47562.

Luk, E., Carroll, M., Baker, M., \& Culotta, V. C. (2003). Manganese activation of superoxide dismutase 2 in Saccharomyces cerevisiae requires MTM1, a membrane of the mitochondrial carrier family. Proceedings of the National Academy of Sciences of the United States of America, 100, 10353-10357.

Luk, E., Yang, M., Jensen, L. T., Bourbonnais, Y., \& Culotta, V. C. (2005). Manganese activation of superoxide dismutase 2 in the mitochondria of Saccharomyces serevisiae. Journal of Biological Chemistry, 280, 22715-22720.

Maia, I. G., Benedetti, C. E., Leite, A., Turcinelli, S. R., Vercesi, A. E., \& Arruda, P. (1998). AtPUMP: an Arabidopsis gene encoding a plant uncoupling mitochondrial protein. FEBS Letters, 429, 403-406.

Marschner, H. (1995). Mineral nutrition of higher plants: 2nd Edition, Academic Press, London.

Meier, B., Barra, D., Bossa, F., Calabrese, L., \& Rotilio, G. (1982). Synthesis of either Fe- or Mn-superoxide dismutase with an apparently identical protein moiety by an anaerobic bacterium dependent on the metal supplied. Journal of Biological Chemistry, 257, 13977-13980.

Millar, A. H., \& Heazlewood, J. L. (2003). Genomic and proteomic analysis of mitochondrial carrier proteins in Arabidopsis. Plant Physiology, 131, 443-453.

Miller, A. F. (2012). Superoxide dismutases: Ancient enzymes and new insights. FEBS Letters, 586, 585-595.

Morgan, M. J., Lehmann, M., Schwarzlander, M., Baxter, C. J., Sienkiewicz-Porzucek, A., Williams, T. C., . . . Finkemeier, I. (2008). Decrease in manganese superoxide dismutase leads to reduced root growth and affects tricarboxylic acid cycle flux and mitochondrial redox homeostasis. Plant Physiology, 147, 101-114.

Murashige, T., \& Skoog, F. (1962). A revised medium for rapid growth and bio assays with tobacco tissue cultures. Physiologia Plantarum, 15, 473-497.

Naranuntarat, A., Jensen, L. T., Panicni, S., Penner-Hahn, J. E., \& Culotta, V. C. (2009). The interaction of mitochondrial iron with manganese superoxide dismutase. Journal of Biological Chemistry, 284, 2263322640 .

Nickelsen, J., \& Rengstl, B. (2013). Photosystem II assembly: From cyanobacteria to plants. Annual Review of Plant Biology, 64, 609-635.

Okada, S., Kanematsu, S., \& Asada, K. (1979). Intracellular distribution of manganese and ferric superoxide dismutases in blue-green algae. FEBS Letters, 103, 106-110.

Palmieri, F., Pierri, C. L., Grassi, A. D., Nunes-Nesi, A., \& Fernie, A. R. (2011). Evolution, structure and function of mitochondrial carriers: a review with new sights. Plant Journal, 66, 161-181.

Picault, N., Hodges, M., Palmieri, L., \& Palmieri, F. (2004). The growing family of mitochondrial carriers in Arabidopsis. Trends in Plant Science, 9, 138-146.

Pilon, M., Ravet, K., \& Tapken, W. (2011). The biogenesis and physiological function of chloroplast superoxide dismutases. Biochimica et Biophysica Acta, 1807, 989-998. 
Portnoy, M. E., Liu, X. F., \& Culotta, V. C. (2000). Saccharomyces cerevisiae expresses three functionally distinct homologues of the Nramp family of metal transporters. Molecular and Cellular Biology, 20, 78937902 .

Pugh, S. Y., \& Fridovich, I. (1985). Induction of superoxide dismutases in Escherichia coli B by metal chelators. Journal of Bacteriology, 162, 196-202.

Rae, T. D., Torres, A. S., Pufahl, R. A., \& O'Halloran, T. V. (2001). Mechanism of Cu,Zn-superoxide dismutase activation by the human metallochaperone hCCS. Journal of Biological Chemistry, 276, 51665176.

Regelsberger, G., Atzenhofer, W., Ruker, F., Peschek, G. A., Jakopitsch, C., Paumann, M., . . . Obinger, C. (2002). Biochemical characterization of a membrane-bound manganese-containing superoxide dismutase from the cyanobacterium Anabaena PCC 7120. Journal of Biological Chemistry, 277, 43615-43622.

Robinson, A. J., \& Kunji, E. R. S. (2006). Mitochondrial carriers in the cytoplasmic state have a common substrate binding site. Proceedings of the National Academy of Sciences of the United States of America, 103, 2617-2622.

Rodríguez-Celma, J., Tsai, Y. H., Wen, T. N., Wu, Y. C., Curie, C., \& Schmidt, W. (2016). Systems-wide analysis of manganese deficiency-induced changes in gene activity of Arabidopsis roots. Scientific Reports, 6,35846 .

Rostami, G. H., \& Ahangar, A. G. (2013). The effect of cow manure application on the Distribution Fractions of $\mathrm{Fe}, \mathrm{Mn}$ and $\mathrm{Zn}$ in agricultural soils. IOSR Journal of Agriculture and Veterinary Science, 6, 60-66.

Schneider, A., Steinberger, I., Herdean, A., Gandini, C., Eisenhut, M., Kurz, S., . . Leister, D. (2016). The evolutionarily conserved protein PHOTOSYNTHESIS AFFECTED MUTANT71 is required for efficient manganese uptake at the thylakoid membrane in Arabidopsis. Plant Cell, 892-910.

Schwab, R., Ossowski, S., Riester, M., Warthmann, N., \& Weigel, D. (2006). Highly specific gene silencing by artificial microRNAs in Arabidopsis. Plant Cell, 18, 1121-1133.

Sevilla, F., López-Gorgé, J., \& del Río, L. A. (1982). Characterization of a manganese superoxide dismutase from the higher plant Pisum sativum. Plant Physiology, 70, 1321-1326.

Slooten, L., Capiau, K., Camp, W. V., Montagu, M. V., Sybesma, C., \& lnzé, D. (1995). Factors affecting the enhancement of oxidative stress tolerance in transgenic tobacco overexpressing manganese superoxide dismutase in the chloroplasts. Plant Physiology, 107, 737-750.

Socha, A. L., \& Guerinot, M. L. (2014). Mn-euvering manganese: the role of transporter gene family members in manganese uptake and mobilization in plants. Frontiers in Plant Science, 5, 106.

Su, Z., Chai, M. F., Lu, P. L., An, R., Chen, J., \& Wang, X. C. (2007). AtMTM1, a novel mitochondrial protein, may be involved in activation of the manganese-containing superoxide dismutase in Arabidopsis. Planta, 226, 1031-1039.

Van Camp, W., Willekens, H., Bowler, C., Van Montagu, M., Inzé, D., Langebartels, C., \& Sandermann, H. (1994). Elevated levels of superoxide dismutase protect transgenic plants against ozone damage. Biotechnology, 12, 165-168.

Vance, C. K., \& Miller, A. F. (2001). Novel insights into the basis for Escherichia coli superoxide dismutase's metal ion specificity from Mn-substituted FeSOD and Its very high E(m). Biochemistry, 40, 13079-13087.

Walter, M., Chaban, C., Schütze, K., Batistic, O., Weckermann, K., Näke, C., . . Kudla, J. (2004). Visualization of protein interactions in living plant cells using bimolecular fluorescence complementation. Plant Journal, 40, 428-438. 
Wang Y, Ying Y, Chen J, Wang X. 2004. Transgenic Arabidopsis overexpressing Mn-SOD enhanced salttolerance. Plant Science 167, 671-677.

Wang, C., Xu, W., Jin, H., Zhang, T., Lai, J., Zhou, X., . . Yang, C. (2016). A putative chloroplast-localized $\mathrm{Ca}(2+) / \mathrm{H}(+)$ antiporter CCHA1 is involved in calcium and $\mathrm{pH}$ homeostasis and required for PSII function in Arabidopsis. Molecular Plant, 9, 1183-1196.

Wang, Y., Ying, Y., Chen, J., \& Wang, X. (2004). Transgenic Arabidopsis overexpressing Mn-SOD enhanced salt-tolerance. Plant Science, 167, 671-677.

Watanabe, A., Nakazono, M., Tsutsumi, N., \& Hirai, A. (1999). AtUCP2: a novel isoform of the mitochondrial uncoupling protein of Arabidopsis thaliana. Plant and Cell Physiology, 40, 1160-1166.

Weigel, D., \& Glazebrook, J. (2002). Arabidopsis: A Laboratory Manual: Cold Spring Harbor, NY: Cold Spring Harbor Laboratory Press.

Wintjens, R., Noël, C., May, A. C. W., Gerbod, D., Dufernez, F., Capron, M., . . . Rooman, M. (2004). Specificity and phenetic relationships of iron- and manganese-containing superoxide dismutases on the basis of structure and sequence comparisons. Journal of Biological Chemistry, 279, 9248-9254.

Witholt, R., Gwiazda, R. H., \& Smith, D. R. (2000). The neurobehavioral effects of sub-chronic manganese exposure in the presence and absence of pre-Parkinsonism. Neurotoxicology and Teratology, 22, 851-861.

Yang, M., Cobine, P. A., Molik, S., Naranuntarat, A., Lill, R., Winge, D. R., \& Culotta, V. C. (2006). The effects of mitochondrial iron homeostasis on cofactor specificity of superoxide dismutase 2. EMBO Journal, $25,1775-1783$.

Yang, T. J., Perry, P. J., Ciani, S., Pandian, S., \& Schmidt, W. (2008). Manganese deficiency alters the patterning and development of root hairs in Arabidopsis. Journal of Experimental Botany, 59, 3453-3464.

Yoo, S. D., Cho, Y. H., \& Sheen, J. (2007). Arabidopsis mesophyll protoplasts: a versatile cell system for transient gene expression analysis. Nature Protocols, 2, 1565-1572.

Zhang, B., Zhang, C., Liu, C., Jing, Y., Wang, Y., Jin, L., . . Luan, S. (2018). Inner envelope CHLOROPLAST MANGANESE TRANSPORTER 1 supports manganese homeostasis and phototrophic growth in Arabidopsis. Molecular Plant, 11, 943-954.

\section{FIGURE LEGENDS}

Figure 1. Mitochondrial localization of AtMTM1 and AtMTM2, and their interactions with AtMSD1. (A) Arabidopsis protoplasts were transfected with AtMTM1-YFP and AtMTM2-YFP to observe mitochondrial localization. (B) The $\mathrm{YFP}^{\mathrm{N}}$ was fused to AtMTM1 and AtMTM2, and the $\mathrm{YFP}^{\mathrm{C}}$ was fused to AtMSD1. Constructs were co-transfected in protoplasts for BiFC assay as indicated. The reconstituted YFP signals were observed by confocal microscopy. MitoTracker staining and chlorophyll autofluorescence were used to identify mitochondria and chloroplasts, respectively. Bars $=20 \mu \mathrm{m}$.

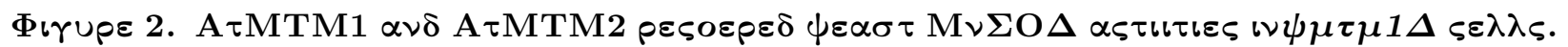
The $\psi \mu \tau \mu 1 \Delta$ cells were transfected withAtMTM1 and AtMTM2, and in-gel SOD activities were analyzed. Yeast CuZnSOD (ySOD1) and MnSOD (ySOD2) activities are indicated by arrowhead and bracket (top), respectively, and immunoblotting used $\alpha$-alcohol dehydrogenase1 (ADH1) antibody(bottom) . ADH1 was an input control.

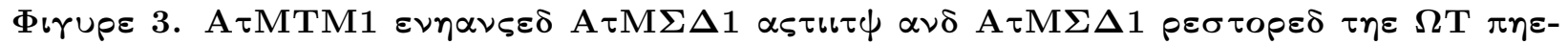

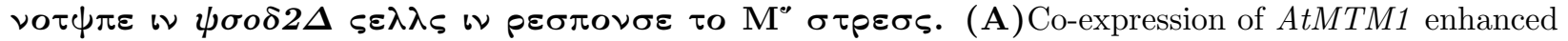
AtMSD1 activity in $\psi \sigma o \delta 2 \Delta$ cells. AtMSD1 activity (top) and protein (bottom) were analyzed. Yeast without (-) and with (+) $100 \mu \mathrm{M} \mathrm{MnSO}_{4}$ supplementation are indicated. Immunoblotting used $\alpha$-AtMSD1 antibody and mitochondrial porin was an input control. (B) AtMSD1 complemented $\psi \sigma o \delta 2 \Delta$ cells to the 
WT phenotype with MV stress. The amount of $5 \mu \mathrm{L}$ diluted culture from $A_{600}=1$ to $10^{-4}$ was grown on YPD medium containing 3 to $5 \mathrm{mM} \mathrm{MV}$.

Figure 4. Expressions of AtMTM1, AtMTM2, and AtMSD1 in various organs. Two to five-weekold organs were used for qPCR analysis. Expression level was normalized relative to the AtMTM1 in root. Data are mean \pm SE of three biological replicates. ${ }^{*}$, significant at $P<0.05$ compared with the root value. AtPP2A was an internal control.

Figure 5. Post-transcriptional regulation of MnSOD and gene expressions of AtMTM1, AtMTM2, and AtMSD1 under MV stress. (A) Two-week-old seedlings were treated without or with 0.1 to $10 \mu \mathrm{M}$ MV for $24 \mathrm{~h}$. An amount of $30 \mu \mathrm{g}$ protein was used for in-gel SOD activity assay (top) and immunoblotting used $\alpha$-AtMSD1 antibody (bottom). Actin was an input control. AtMSD1 activity and protein were normalized relative to the control without MV treatment. (B) Two-week-old seedlings were treated without (-, control) or with $5 \mu \mathrm{M}$ MV for 2 to $12 \mathrm{~h}$. Expression level was normalized relative to the control of AtMTM1 or AtAOX1A. The oxidation-responsive gene of mitochondrial AtAOX1A was used as a reference. Data are mean $\pm \mathrm{SE}$ of three biological replicates. ${ }^{*}$, significant at $P<0.05$ compared with the control.AtPPQA was an internal control.

Figure 6. Expressions of AtMTM1, AtMTM2, and AtMSD1 in response to different metal treatments. Two-week-old seedlings were treated without (-; control) or with $100 \mu \mathrm{M}$ metal ions of $\mathrm{MnCl}_{2}$, Fe citrate, $\mathrm{CuSO}_{4}, \mathrm{ZnSO}_{4}, \mathrm{MgCl}_{2}$, and $\mathrm{CaCl}_{2}$ for $24 \mathrm{~h}$. Expression level was normalized relative to the control. Data are mean $\pm \mathrm{SE}$ of three biological replicates. ${ }^{*}$, significant at $P<0.05$ compared with the control. AtPP2A was an internal control.

Figure 7. Expressions of AtMTM2 in mtm1-i and AtMTM1 in mtm2 mutants in adapting to a shock of MV stress. Two-week-old seedlings were treated without (control) or with 1 to $10 \mathrm{nM} \mathrm{MV}$ for $12 \mathrm{~h}$. (A and B) AtMTM2expression in mtm1-i mutant and AtMTM1 expression inmtm2 mutant were analyzed by qPCR, respectively. Expression level was normalized relative to the control of WT. Data are mean $\pm \mathrm{SE}$ of three biological replicates. ${ }^{*}$, significant at $P<0.05$ compared with the WT. AtPP2A was an internal control.

Figure 8. Root lengths of $m t m 1-i$ and $m t m 2$ mutants in adaption to long-term MV stress. (A and B) Root lengths of $m t m 1-i$ seedlings grown on 1 to $50 \mathrm{nM} \mathrm{MV}$ for $8 \mathrm{~d}$ and mtm2on 0.5 to $10 \mathrm{nM} \mathrm{MV}$ for $10 \mathrm{~d}$ were measured, respectively. Data are mean $\pm \mathrm{SE}$ of three biological replicates. ${ }^{*}$, significant at $P<$ 0.05 compared with the WT.

Figure 9. Characterization of $\boldsymbol{m t m 1 - i} \mathbf{m t m} 2$-double mutant. (A) Expression level was normalized relative to the WT. AtPP2Awas an internal control. (B) Mn and FeSOD activities in six siblings of mtm1-i mtm2-\#1 line (top). Coomassie blue staining gel showed the ribulose bisphosphate carboxylase large subunit $(\mathrm{RbcL})$ that was used as an input control (bottom) . (C)Mn and FeSOD activity of three independent plants was normalized relative to the WT, respectively. Data are mean $\pm \mathrm{SE}$ of three biological replicates. *, significant at $P<0.05$ compared with the WT. (D) Early-flowering phenotype of one-month-oldmtm1-i mtm2-\#1 plants.

Figure 10. Root lengths of $m t m 1-i, m t m 2$, and $m t m 1-i$ mtm2-double mutants under Mn supplementation. WT and three mutants grown on $1 / 2 \mathrm{MS}$ basal medium supplemented without (-) or with additional 10, 50, and $500 \mu \mathrm{M} \mathrm{MnCl}_{2}$ for $6 \mathrm{~d}$ and root lengths were measured. Data are mean $\pm \mathrm{SE}$ of three biological repeats. ${ }^{*}$, significant at $P<0.05$ compared with the WT.

Figure 11. Subcellular localization and SOD activity of modified AtMSD1. (A) Localizations of mitochondrial AtMSD1, cytosol-destined $\Delta$-TP-AtMSD1, and chloroplast-destined Chl-TP-AtMSD1 were analyzed by confocal microscopy. YFP was fused to the C-terminal end of each tester. Bar $=20 \mu \mathrm{m}$. (B)

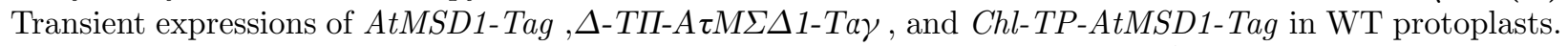
Transfections without (-) or with (+) $30 \mu \mathrm{g}$ plasmid DNA of AtMSD1-Tag in 106 protoplasts are indicated. In-gel SOD activity assay (top) and immunoblotting with $\alpha$-AtMSD1 and $\alpha$-Actin antibodies (bottom) 
were conducted. Actin was an input control.

Figure 12. Exogenous expression of AtMSD1-3xFLAG in WT and mtm1-i mtm2 mutant protoplasts. Transfections with 5 and $10 \mu \mathrm{g}$ plasmid DNA in $10^{6}$ protoplasts are indicated. In-gel SOD activity assay (top) and immunoblotting with $\alpha$-FLAG and $\alpha$-Actin antibodies (bottom) were conducted. Actin was an input control.

Figure 13. Mn and Fe contents in mtm1-i, mtm2, andmtm1-i mtm2 mutants under $\mathrm{MnCl}_{2}$ treatment. Two-week-old seedlings were incubated without (control) or with $100 \mu \mathrm{M} \mathrm{MnCl} 2$ for $24 \mathrm{~h}$. (A and $\mathbf{B}) \mathrm{Mn}$ and Fe contents in root and shoot were measured by ICP-OES, respectively. The index of metal retention ability represents the ratio of ion content in the Mn treatment to the control. Data are mean \pm SE of three biological repeats. ${ }^{*}$, significant at $P<0.05$ compared with the WT.

\section{Hosted file}

PCE-Submittion-Figures All-Hu (final).pdf available at https://authorea.com/users/334466/ articles/460430-mitochondrial-atmtm1-and-atmtm2-are-required-for-mnsod-activationstress-response-and-ion-homeostasis 
Figure 1
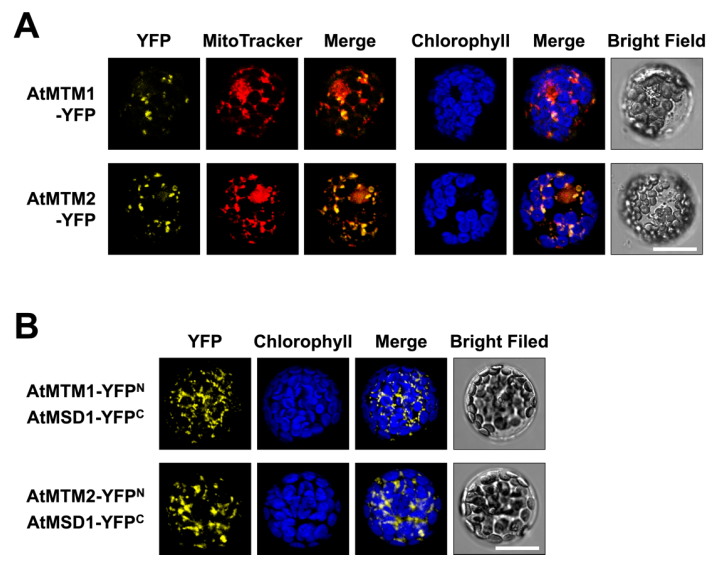

Figure 1. Mitochondrial localization of AtMTM1 and AtMTM2, and their interactions with AtMSD1. (A) Arabidopsis protoplasts were transfected with AtMTM1-YFP and AtMTM2-YFP to observe mitochondrial localization. (B) The YFP $^{\mathrm{N}}$ was fused to AtMTM1 and AtMTM2, and the $\mathrm{YFP}^{\mathrm{C}}$ was fused to AtMSD1. Constructs were co-transfected in protoplasts for BiFC assay as indicated. The reconstituted YFP signals were observed by confocal microscopy. MitoTracker staining and chlorophyll autofluorescence were used to identify mitochondria and chloroplasts, respectively. Bars $=20 \mu \mathrm{m}$. 